\title{
TIME
}

B R I L L

Timing \& Time Perception 5 (2017) 297-327

$\overline{\text { brill.com/time }}$

Review

\section{The Development of Temporal Concepts: Learning to Locate Events in Time}

\author{
Teresa McCormack ${ }^{1, *}$ and Christoph Hoerl ${ }^{2}$ \\ 1School of Psychology, Queen's University Belfast, Belfast, Northern Ireland, UK \\ ${ }^{2}$ Department of Philosophy, University of Warwick, Coventry, UK
}

Received February 2017; accepted April 2017

\begin{abstract}
A new model of the development of temporal concepts is described that assumes that there are substantial changes in how children think about time in the early years. It is argued that there is a shift from understanding time in an event-dependent way to an event-independent understanding of time. Early in development, very young children are unable to think about locations in time independently of the events that occur at those locations. It is only with development that children begin to have a proper grasp of the distinction between past, present, and future, and represent time as linear and unidirectional. The model assumes that although children aged two to three years may categorize events differently depending on whether they lie in the past or the future, they may not be able to understand that whether an event is in the future or in the past is something that changes as time passes and varies with temporal perspective. Around four to five years, children understand how causality operates in time, and can grasp the systematic relations that obtain between different locations in time, which provides the basis for acquiring the conventional clock and calendar system.
\end{abstract}

\section{Keywords}

Time perception, interval timing, temporal frameworks, tense, temporal-causal reasoning, eventindependent thought about time

\section{Introduction}

A basic distinction can be made between two ways in which time features in people's mental lives: as a dimension of things that unfold in time, and as a framework within which those things can be located. Psychological studies of the former focus on people's ability to process and represent information about the amount of time that events last for. However, events do not just have specific durations,

* To whom correspondence should be addressed. E-mail: t.mccormack@qub.ac.uk 
they also have temporal locations, just as objects do not only have spatial extent, they also have spatial locations. Thus, we can talk about temporal frameworks systems for representing temporal locations - in the same way as we can talk about spatial frameworks (McCormack \& Hoerl, 1999). The most familiar temporal framework that we operate with is the conventional clock and calendar system. However, the clock and calendar system is mastered relatively late in development, meaning that in characterizing the acquisition of mature temporal cognition, we need to consider how children represent temporal locations before they master this system. Much existing developmental research on time has focused on children's ability to process and represent duration information. In this paper, we will instead focus primarily on the development of the ability to locate events in time, and we make a preliminary attempt to provide a model of developmental changes in this ability. Before doing so, we briefly summarize some existing work on time in children.

\subsection{Time as a Dimension: Processing Duration}

It is clear that children, from an early age, are sensitive to how long events last for. A series of innovative studies of timing in infancy have demonstrated infants' sensitivity to the duration of events and the developmental continuity of the processes involved in time perception (e.g., Brannon et al., 2004; Colombo \& Richman, 2002; VanMarle \& Wynn, 2006). Moreover, research with older children, much of which has been conducted by Sylvie Droit-Volet, has carefully charted developmental improvements in sensitivity to duration and also identified some of the mechanisms that contribute to such improvements (see Droit-Volet, 2013, 2016, for review). However, as Droit-Volet has also argued, being sensitive to the duration of events, which is present even in infancy, is not the same thing as understanding that events have particular durations (Droit-Volet \& Rattat, 1999; Rattat \& Droit-Volet, 2007). Sensitivity to durations can be manifested by children adjusting their behavior appropriately to the durations of events that they perceive and process. However, children can adjust their behavior appropriately to event duration without an explicit grasp of the idea that events have durations. Thus, we can ask when children possess the concept of duration itself.

Historically, research on this issue was conducted within the Piagetian tradition (stemming from Piaget, 1969). Research in this tradition takes as its starting point the idea that having a notion of temporal duration is a matter of being able to coordinate the relations between the dimensions of time, speed, and distance in the movement of objects (what Piaget thought of as the Newtonian notion of time). Empirical work primarily focused on the nature of the errors children made in their duration judgments that suggested a conflation of these dimensions. While this work undoubtedly highlighted some striking errors in children's timing judgments, this tradition has generated little research interest since the 1980s. This is largely because researchers have come to reject some of the 
central tenets of this approach. Fully coordinating the dimensions of speed, distance, and time is a complex achievement, and even 8- to 12-year-olds can struggle to make accurate judgments of duration when these dimensions are confounded (Richards, 1982; Siegler \& Richards, 1979). It has therefore been argued that there must be more basic aspects of the mature notion of time that this approach neglects (Levin, 1982, 1992; Weist, 1989; Wilkening, 1982). Moreover, the idea that we can measure children's notion of time primarily by looking at the accuracy of their duration judgments, as is assumed in the Piagetian approach, also faces the problem that even the duration judgments of adults can be affected by irrelevant stimulus dimensions (see Matthews \& Meck, 2016, for a recent review), whilst, at the same time, even relatively young children can make some judgments of duration, arguably without relying on the sort of complex inferential processes highlighted within the Piagetian tradition (Droit-Volet, 2002, 2013; Droit-Volet \& Coull, 2016).

Weist (1989) has spoken of a Piagetian void' that this approach leaves, even setting aside other problems it faces, because it has little to say about early childhood. It is this void that we hope to make some initial steps to fill, and we think the void is not just one regarding the early years, but also a void regarding central aspects of temporal cognition and their development. The Piagetian tradition focuses on time as a dimension of events - i.e., as lasting for a duration of a specific magnitude - with this approach trying to identify when children can separate out this dimension from other stimulus dimensions. Although the Piagetian approach has largely been discarded, recent research has retained this focus on time as a dimension. For instance, a central question in recent research on temporal representation has been whether children can make systematic mappings between temporal duration and other dimensions (Lourenco \& Longo, 2010; Srinivasan \& Carey, 2010). And, as mentioned above, most of the developmental research on time has continued to concentrate on changes in the accuracy with which children make judgments about duration. While these issues are clearly fundamental ones, here we want to emphasize the other, equally important, way in which time features in cognition. As we have already pointed out, time is also used as a framework insofar as events are located within time (McCormack \& Hoerl, 1999): adults think of events as positioned somewhere in the past, present, or future and also as having locations in time relative to each other, with there being systematic before-and-after relations between these locations. We take it to be a crucial, but underexplored, developmental question whether children operate with the same sort of temporal framework as adults, or whether there are more developmentally basic ways of representing the locations of events.

While we will be focusing on children's temporal frameworks, we also acknowledge that the question framed above and addressed by Piaget - i.e., when do children have a concept of duration - remains an important question. Moreover, introducing the issue of the acquisition of a mature temporal framework raises a 
further question of how this developmental process is linked to the acquisition of a concept of duration. Are these separable developmental achievements, or are they linked in important ways? Part of the power of our conventional temporal framework, the clock and calendar system, is that it deals both with duration and with temporal location: e.g., days, weeks, and years last for a certain duration, but, by assigning a time and date to an event, its time of occurrence is also located relative to other times. Thus, mature temporal cognition integrates both these aspects of time. We will return to the issue of how this is achieved developmentally towards the end of the paper.

\subsection{What are the Properties of Mature Temporal Cognition?}

There are some fundamental questions about the way children think about time that we do not as yet have the answers for. For example, we do not know whether in locating events in time, children make use of the basic distinction between past, present, and future that adults operate with. This lack of knowledge stems from both a methodological and a theoretical gap in the literature on cognitive development. With regard to methodology, unlike in some areas of developmental psychology, there is no agreed set of tasks that are used to tap children's temporal concepts. This methodological gap stems at least in part from the theoretical gap: there is no agreed theoretical approach that actually specifies the core features of mature temporal understanding from which such tasks might be derived. For this reason, we start by outlining three core features of adults' mature concept of time that will shape our discussion of the developmental issues.

(i) Time is represented as linear and unidirectional. A mature temporal framework locates events on a time line stretching back into the past and forward into the future, with each location on the line being unique and non-reoccurring. This time line has a direction in so far as events successively become present in the future direction, as expressed in the idea of the 'flow' or 'passage' of time.

(ii) Time is unified. A single time line is used to represent temporal locations, and, as a result of this, every point in time stands in a systematic before/after relation to every other point in time. Transitivity holds between these relations: if a point in time $\mathrm{A}$ is before $\mathrm{B}$ and $\mathrm{B}$ is before $\mathrm{C}$, then $\mathrm{A}$ is also before $\mathrm{C}$.

(iii) Time is event independent. Although time is populated by events, adults are able to think of points in time independently of the particular events that occur at them. Being able to distinguish between a point in time and any events that might occur at that point is an important aspect of mature temporal cognition. This aspect of temporal cognition is most clearly embodied in the clock and calendar system, which can be used to specify any point in time without making reference to any events that have occurred or might occur at that temporal location. However, we are not claiming that children only have this way of thinking about time once they have mastered the clock and calendar system. Rather this way of thinking about time is likely to be a developmental prerequisite to being 
able to begin to learn such a system, and may be intact long before children master it (which they only do relatively late in development).

Note that our aim here is to characterize features of everyday thinking about time. Science, e.g., in the form of the theory of relativity, provides a somewhat more complex picture of the nature of reality itself, which reveals some of the assumptions listed above to be in need of qualification. Similarly, some philosophers working on the metaphysics of time question the extent to which some of the assumptions we have listed correctly reflect the nature of time itself, or are ultimately illusory. (On both of these issues, see, e.g., Dainton, 2010.) However, we take it as fairly uncontroversial that the descriptions given above capture three core features of the way in which we do in fact think of or speak about time in ordinary, everyday contexts. The purpose of specifying these core features is to provide a template against which to compare children's ways of thinking about and representing time: we can ask at what point in development children possess a notion of time that has these features, and raise the possibility that early in development the way in which children represent time may lack one or more of them. That is, it is possible that young children do not think of time as linear and unidirectional, do not have a unified way of representing the temporal locations of events, and cannot think of time in an event-independent way.

\subsection{Temporal Perspective-Taking: A Hallmark of Mature Temporal Cognition}

Before describing our developmental model, we want to further elaborate the properties of the type of mature temporal cognition that we take to be the endpoint of development. In the previous section, we outlined the features of the notion of time that we wish to focus on. Here, we want to stress that a hallmark of possessing such a notion is the ability to engage in what can be termed temporal perspective taking, an ability that we will now describe.

Part of what it is to possess a linear and unidirectional notion of time is to understand that what points in time are in the past, present, and future is not fixed. Rather, it changes systematically as time goes on. That is, for any ordered set of times $A, B$, and $C$ that have now passed, there was a point in time at which what was to happen at $A, B$, and $C$ was still in the future. Similarly, from the perspective in time at which $A$ has just passed, $A$ is in the past, whereas $B$ and $C$ are still in the future and so on. One way to describe this is to say that a mature notion of time supports a type of temporal perspective-taking that allows one to consider which times are in the past and which in the future from points in time other than the point one currently occupies (e.g., from the temporal perspective of yesterday, today was still in the future, but today will be in the past from the temporal perspective of tomorrow).

The idea that temporal perspective-taking is a hallmark of a mature notion of time is not new, and has been put forward by other theorists (Cromer, 1971; Harner, 1982; Weist, 1989; see also McCormack, 2014; McCormack \& Hoerl, 1999), but 
we want to emphasize here a particularly crucial aspect of temporal perspectivetaking, namely that because time is understood as unidirectional, temporal perspective-taking must be grounded in a grasp of the asymmetry between past and future times. In people's everyday understanding of time, there is a basic asymmetry between the past and the future: thinking about times as being in the past' involves thinking of them in a quite different way than thinking about them as being 'in the future' does. One way to capture this asymmetry in the everyday understanding of time is in terms of the idea that facts about what happened in the past are not alterable, whereas facts about what will happen in the future are at least potentially alterable. ${ }^{1}$ We argue that mature temporal perspective-taking must involve putting to work this idea, and it is only because it has this element that it embodies a grasp of the unique directionality of time.

To make this clear, it is worth considering what is distinctive about temporal perspective-taking that makes it a quite different sort of cognitive achievement from spatial perspective-taking. In spatial perspective-taking, one may have to grasp that, for example, from this perspective in space the table is in front and the chair is behind one, whereas from another perspective both of these are behind one. In doing so, of course, one must be able to distinguish in some way between 'in front' and 'behind'. However, although whether something is in front of or behind one has implications for one's actions, there is nothing inherently different about the nature of those actual locations in space and indeed one could change whether something is in front of or behind one freely by moving to a different place. The temporal case is quite different. Whether something is in the past or in the future is not something that is in our power to determine, and which of two temporal locations is in the past and which is in the future is not something that can be reversed: we might say that in the everyday understanding of time there is something ontologically different about locations in the past and the future. Our suggestion is that temporal perspective-taking underpinned by a mature grasp of time must involve understanding this, which is why we describe it as perspectivetaking that involves putting to work the idea that facts about what happened in the past are unalterable whereas facts about what will happen in the future are still potentially alterable. An important developmental question, therefore, is when children can engage in this sort of perspective-taking.

1 We will be using this way of talking to characterize the kind of idea of the future as 'open' expressed in sentences like: "I was going to go to the cinema this evening, but now I think I might stay at home." Again, note that this is meant to capture an aspect of our everyday thinking about time and events in time. Whether the future is genuinely different from the past in reality is a question on which there is a great deal of debate in philosophy, because it is of course equally as true of future times as of past ones that they can only ever contain one of these types of event or the other. (I either will go to the cinema, or I will stay at home). 
We will finish this sub-section by making it clear exactly why we think this sort of temporal perspective-taking involves event-independent thought about time. Consider the case again of an ordered series of times A, B, and C that are now in the past. Someone capable of temporal perspective-taking understands that from the perspective of $\mathrm{A}$, facts about events at $\mathrm{B}$ and $\mathrm{C}$, which have now already taken place, were in fact still potentially alterable. Grasping this means that they understand that, from that perspective, it was still possible for things to unfold differently to how they actually did unfold. That is, they understand that B and C are slots in time that could have been filled with different events than the events that actually did happen. Grasping this means thinking about $\mathrm{B}$ and $\mathrm{C}$ in an eventindependent way: as times that are separable from the events that actually did happen. Note that in spelling things out in this way, we are making a strong connection between being able to think counterfactually and a mature notion of time. Moreover, someone who can think in this way can also understand that there may be events that happened at A that have left no observable traces in the present, sometimes taken to be crucial element of a proper understanding what it is for an event to be in the past (Hoerl, 2007). This is because they understand that while what happened at A may have left its traces on how things were at a subsequent time $B$, facts about what would happen subsequent to $B$ were still alterable when $B$ was present, so that things happening at or after B may have removed any such traces again.

\subsection{Culture and Notions of Time}

In proposing a developmental model of the acquisition of a notion of time that has the features we have emphasized, we need to briefly consider to what extent such a model is likely to capture something universal, rather than culture-specific. That is to say, are there cultures in which the features of mature temporal cognition that we have outlined are not in fact found? Probably the key controversial issue here is whether all cultures operate with a linear notion of time. We note that a linear notion of time, according to which temporal locations are represented as unique and non-recurring, could be instantiated in a number of different ways in the spatialized representations of time that are often referred to as 'mental time lines' (Bender \& Beller, 2014). In a number of cultures, including English-speaking Western cultures, time lines seem to have particular axes: time is spatialized either on the sagittal axis, with the past behind and the future in front relative to the body, or the transversal axis with the past to the left and future to the right. As is now well-established, other cultures spatialize times in different ways. In some instances, this involves a reversal of the assignment of past/future to portions of the sagittal or transversal axes (Fuhrman \& Boroditsky, 2010; Núñez \& Sweetser, 2006) but it may involve use of a different axis or another spatial framework: Mandarin speakers make use of a vertical axis (Boroditsky et al., 2011), and Boroditsky 
and Gaby (2010) describe an Aboriginal tribe that represent time as running East to West.

Despite these cross-cultural differences, all of these ways of spatializing time can be interpreted just as different ways of spatially instantiating a linear notion of time; what varies is the nature of the instantiation or in some instances the type of spatial metaphor used (e.g., the course of a river or the inclination of a landscape; see Bender \& Beller, 2014). The trickier question is whether there are any cultures in which the notion of linearity itself is entirely absent. That is, are there any cultures in which people do not think of times as unique and non-recurrent? A culture that represented times only in a cyclical or repeating temporal framework would be of this sort. While some existing or past cultures clearly do/did emphasize cyclical or repeating patterns in time more than others (Aveni, 2000; Le Guen et al., 2012; Sinha \& Gärdenfors, 2014), what is highly controversial is whether this is/was the only notion of time available to them (Gell, 1992; Hassig, 2001). It is beyond the scope of this paper to properly address this question, though we note that looking at the characteristics of culturally specific spatial metaphors of time or calendar systems is not the only way to examine this issue. For example, Campbell (1997) has argued that a notion of time as linear is typically employed in constructing autobiographical narratives to describe events in one's life (see also Hoerl \& McCormack, 2005); if he is correct, then it seems unlikely that there is a culture that entirely lacks this mode of thinking about time (see McCormack, 2015, for further discussion,).

\section{A Developmental Model of the Acquisition of Temporal Concepts}

In this section, we propose a model of children's acquisition of the notion of time we have described in Section 1.2, i.e., the notion of time that underpins the temporal framework used by adults to locate events. This model does not address all aspects of the acquisition of temporal concepts; most notably it does not consider the acquisition of a concept of duration. The developmental endpoint that the model aims to capture is the notion of time that has the properties described in the previous subsection. Thus, the model holds that more developmentally primitive ways of thinking about time lack some of the core features of mature temporal cognition we have outlined, and that younger children are not capable of the type of temporal perspective-taking described in Section 1.3.

One difficulty in proposing any comprehensive developmental model in this area is the lack of data from studies that have directly addressed key issues about children's notion of time. In putting together our model, we have drawn on existing data from a variety of sources, including research on developmental psycholinguistics, studies of children's memory, planning, and future thinking, and experiments specifically addressing particular aspects of children's reasoning about time and about causation. It should be emphasized that we have used the 
principle of assuming only the minimal level of understanding necessary to explain what is currently known about children's cognition at a specific age; this model is thus conservative in what abilities it ascribes to young children, and perhaps may prove to be unnecessarily conservative. Moreover, we recognize that there is still a large empirical gap to be filled; the account we provide is speculative and can only act as a springboard for further research in this area.

Figure 1 shows diagrammatically the different developmental stages that we are proposing. The figure shows how children represent events or times that (objectively) are in the past and the future at each of four stages. Before describing

a)

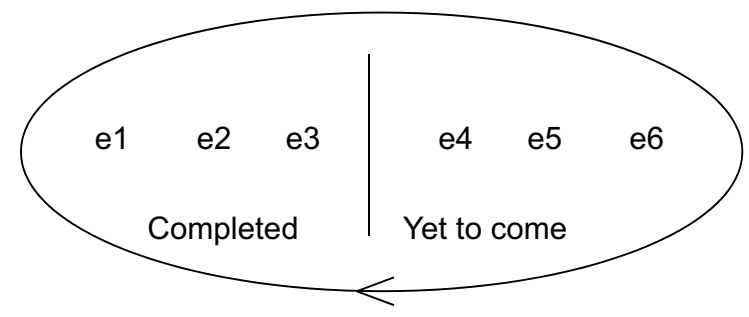

Representations of repeated event sequences (<24 months)

e1

b)

c)

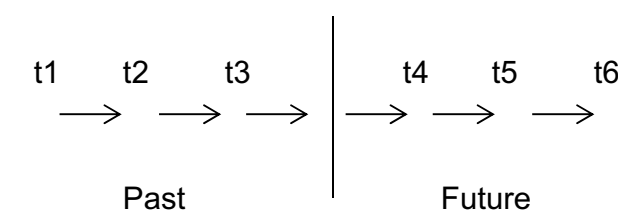

$\mathrm{t} 1 \quad \mathrm{t} 2$

d) e5 e3 e2 Not alterable
Alterable Future e4 e6

Event-based time (2-3 years)
Linear eventindependent time (4-5 years) t4 t5 t6

Future
Abstract time (> 5 years)

Past

\section{Current location \\ in time}

Figure 1. Summary of the developmental model. At Stage (a), representations are of repeated event sequences (as indicated by the circle) and time is not linear. At Stage (b), representations are still of events rather than of times per se, and there is no linear organization. At Stage (c), times are represented and organized linearly underpinned by an understanding of how causation operates in time. At Stage (d), time is represented in a fully abstract and event-independent way. 
each stage in detail, some general points can be made about the developmental shifts that the model tries to capture. First, children are assumed to shift from only being able to represent events [Stages (a) and (b)] to being able to represent points in time [Stages (c) and (d)]. That is, we are assuming that there is a shift from event-dependent to event-independent thought about time; our suggestion is also that whether thought about time is event-independent is a matter of degree and that the model documents a series of changes towards fully event-independent thought about time. Second, it is only Stages (c) and (d) that children are assumed to be able to think about the past and the future per se; at the early stages children treat past events differently compared to those in the future, but are not thinking of these events as located in a different time period, conceived of as such. Third, in Stages (a) and (b), time is not represented linearly and it is not assumed that children grasp the transitivity of the before-and-after relations between events. Thus, at the early stages of development, children's notions of time lack the core features that we have emphasized as characteristic of mature temporal concepts.

This model is intended to capture key changes in the way children think about time that affect how they temporally locate events. Broadly speaking, children change from only being able to represent where in time an event is in terms of its position within an ordered repeated sequence of familiar events, to being able to assign events unique locations in time, and being able to think about whether those locations are currently in the past, present, or future. This emerging understanding then allows children to begin to acquire the conventional clock and calendar system. The first stage of our model begins when children are toddlers, starting to demonstrate in their actions that they are representing certain behavioral routines, and beginning to acquire language. Note that we do not discuss here how younger infants temporally locate events. Very young infants are sensitive to some temporal features of stimuli such as duration and rhythm, and this extends even to neonates (de Hevia et al., 2014; Nazzi et al., 1998), but it is extremely difficult to examine how they locate events in time.

\subsection{Stage (a): Representations of Repeated Event Sequences (18-24 months)}

A basic distinction that can be made between different types of ways of being oriented in time is in terms of whether they involve representations of repeatedly occurring cycles/sequences of events or representations of particular unique times (Campbell, 1995). Recurring event cycles or event sequences of various lengths are ubiquitous in everyday life, and there are reliable temporal relations between locations in such event cycles/sequences. For example, brushing of teeth happens after supper but before bedtime in the evening routine, Tuesday happens before Wednesday but after Monday in the weekly cycle of days, and summer happens after spring but before winter in the annual cycle. Recognizing where events happen within such repeated cycles or event sequences is very important, because it allows actions (e.g., the bedtime routine) to be carried out in the appropriate 
order and supports reliable prediction of what is going to happen next. Notably, though, mature thinkers can not only represent locations within such repeating event cycles/sequences in the right order, they can also think of any given occurrence of an event within a particular cycle or sequence as having a unique temporal location. To give two examples, although the action sequence in the bedtime routine is the same every day, today's tooth brushing occurs at a particular time that is never revisited; similarly, Tuesday always comes before Wednesday but Tuesday $23^{\text {rd }}$ August 2016 is also a unique point in time and in that respect differs from every other Tuesday. Mature thinkers can represent both these unique temporal locations alongside understanding where in repeated event cycle/sequence that type of event happens with respect to other events in the cycle/sequence.

An assumption underpinning our model is that representing locations within repeated event cycles/sequences is more primitive than representing particular times. In fact, we believe that it is more primitive both ontogenetically and phylogenetically; specifically, we have argued elsewhere that there is no reason to assume that animals can think about particular times (Hoerl, 2008; McCormack, 2001; McCormack \& Hoerl, 2011; see also Campbell, 1995). Part of this argument hinges on what the function is of representing temporal locations as particular times; for many types of behavior what matters is knowing the appropriate order in which to carry out an action sequence, and knowing what to expect at any given point in a repeated event cycle/sequence. In fact, it is by no means straightforward to identify non-linguistic behavior that depends upon on a concept of times as unique and unrepeatable (Bennett, 1989; Campbell, 1995, 2006; McCormack \& Hoerl, 2011).

It is evident from relatively early in development that children are adept at learning repeated event sequences (reviewed by Nelson, 1996). Toddlers rapidly acquire behavioral expectations regarding repeated sequences (e.g., expecting to have a bath before sleep time), and parents typically try to shape toddlers' behavior so that they will learn to carry out some action sequences in the appropriate order (e.g., we put on our socks before our shoes). In our model, we characterize this sensitivity as Stage (a), with the oval around the events intended to indicate the repeating nature of the sequences, thus represented, in contrast to the way mature thinkers represent events as happening at unique, non-reoccurring locations on a time line.

There are three important things to stress about the limitations we assume at this stage. First, we see no reason to posit that very young children who can represent locations with respect to repeated event sequences can think about those locations in an event independent way. For example, with respect to the bedtime routine, although there is a sense in which children know 'when' their bath happens (after supper and before bed), there is nothing about this that compels us to assume that they can think about that temporal location as anything other than the point at which bath time happens. In this sense, very young children need not 
be thinking about times per se, but rather their thought is restricted to events that fill time. It is in this strong sense that we assume that earliest notions of time are event-dependent. Second, if all that very young children are able to do is represent and orient themselves within repeated event sequences, there is no reason to believe that they can think about events as located in the past or in the future. A key part of what it is to represent an event as in the past is to represent it as happening at a particular time that can never be revisited. By definition, if children can only represent repeated event sequences, they will not be able to represent events as having happened at a time that can never be revisited. They will have no way of thinking of yesterday's bath time as different from today's or tomorrow's bath time: in all these instances, bath time is represented just in terms of where it falls relative to other events in the bedtime event sequence.

Although we believe this is the case, it should be stressed that in order for children to make use of representations of repeated event sequences, they need to be able to know where they are within an unfolding sequence. That is, they need to be able to use their representation to orient themselves in some way so that they have expectations about what will happen next (e.g., putting on of pyjamas happens next), and can assume that events that have already happened are now over (e.g., once pyjamas are on, it would be very surprising to be given a bath). One way of capturing this is by saying that, at this stage, children need to be capable of representing some events as 'completed' and some events as 'yet to come'. Importantly, though, orienting themselves with respect to events in this way is not the same thing as thinking of events as being located in a past or future time period: in thinking in this way, children are thinking about the status of events (completed/ not completed) rather than the location of points in time (past/future).

Finally, it should be pointed out that if children orient themselves only with respect to repeated event sequences, there is no reason to assume that they have what we have termed a unified way of representing time. That is, they have no single way of representing any given location in time. For example, they may be sensitive to the fact that having a bath happens before going to bed, or that they get into the car before arriving at nursery, but may have no way of thinking about how the daily event of getting into the car is related to the event of having a bath. Thus, although Fig. 1a shows an ordered sequence of events, this should be interpreted as representing a single type of event sequence; there is no reason to assume that very young children can systematically relate ordered sequences to each other.

We speculate that Stage (a) describes children's temporal representations for approximately the first 18-24 months of life. It should be noted, though, that toward the end of this period some children begin to use the past tense for the first time (for examples, see Shirai \& Andersen, 1995; Shirai \& Miyata, 2006; Weist, 1989). The issue then, is how we should interpret early use of the past tense, and whether this early use suggests that very young children do not have the conceptual limitations that we are proposing here. Historically, this has been a highly 
controversial issue (Antinucci \& Miller, 1976; Bronckart \& Sinclair, 1973; Weist, $1989,2014)$, and it is beyond the scope of this paper to summarize the developmental psycholinguistics debate. Detailed analyses suggest that children's first uses of the past tense (Sachs, 1983; Shirai \& Miyata, 2006) are to the immediate rather than the remote past, consistent with the idea that very young children's first use of the past tense is to mark something about the nature of the events themselves (i.e., that they are completed). ${ }^{2}$ In suggesting this, we are not claiming that these very young children cannot represent anything except the here-and-now. We are assuming that young children have the sort of representational capacities widely attributed to them in the post-Piagetian era of cognitive developmental psychology, as demonstrated, e.g., by studies of deferred imitation of event sequences (Bauer \& Wewerka, 1995; Bauer et al., 1994; Meltzoff, 1995) or by the fact that children of this age will refer to absent objects or people (Sachs, 1983). These capacities, though, do not demonstrate that they can think of events as being non-current, but located at another time, in the past.

\subsection{Stage (b): Event-Based Time (2-3 years)}

At this next stage, we assume that children can do more than simply represent locations with respect to repeated event sequences. Between two and three years of age, children typically use both past and future tense and start to use temporal adverbs to make reference to temporally distant events. Studies of children's autobiographical memory consistently suggest that children of this age can provide at least some details of remote past events, and can use the past tense to do so (e.g., Peterson, 2002; Peterson \& Rideout, 1998; Weist \& Zevenbergen, 2008). By the time children are three, they can provide (albeit often sparse) information about events due to occur at some point in the future using the appropriate tense (e.g., Hayne et al., 2011; Quon \& Atance, 2010). Even if children's descriptions of past and future events are often both highly scaffolded by adults' questioning and piecemeal in nature (Fivush \& Nelson, 2004; Nelson \& Fivush, 2004), these findings suggest that children in this age range can do more than simply think about the locations of events in repeated event sequences, meaning that their temporal cognition differs fundamentally from that of younger children.

However, we believe that there may still be important limitations in children's grasp of temporal concepts at this stage. As can be seen in Fig. 1b, our suggestion is that children of this age may still be thinking about events rather than points in time even when considering things that have happened outside the immediate past or present. Moreover, we have characterized them as not having

2 Note that we are not suggesting here that very young children only use the past tense for actions that have a natural end-point or point of achievement. Actions that are not of this nature can still be thought of as completed. 
a linear notion of time that would allow them to systematically represent the before-and-after relations between events. And because children are assumed not to be representing the same domain of times as mature thinkers, we do not assume that they have full-blown concepts of the past and future either. Children of this age use tense appropriately, indicating that they draw an important distinction between two categories of events. However, what is at issue is whether these two categories map on to the notions of past and future, as they are usually understood. In our model, we suggest that although children of this age draw a clear distinction between past and future events, it need only be assumed that they think of these events as having different statuses, rather than occurring on different parts of a time line that centres on the present. It is because of this that we still consider thought about time to be event-dependent at this age.

We pointed out in Section 1.3 that in adults' everyday understanding of time, there is an asymmetry between the past and the future, and that part of this asymmetry is that things in the past are unalterable, whereas things in the future are yet to be decided. Our suggestion is that children's earliest grasp of the difference between the past and future may consist primarily in them categorizing past and future events differently, i.e., they think of facts about past events as unalterable and facts about future events as (at least potentially) alterable, and it is this sense that the events fall into two different categories. Using these two categories appropriately is very important - for example, so as not to miss out on the opportunity to change things that still can be changed by dwelling too much on things that can no longer be changed - but in itself falls short of fully grasping the distinction between past and future times. The suggestion is that children may operate with this categorical distinction between events without having a linear, unified, eventindependent notion of time.

In suggesting these limitations in two- to three-year-olds' notion of time, one key consideration is whether children have any grasp of how temporally distant events are related to each other. What is undoubtedly true is that children of this age can sometimes provide temporally ordered descriptions of an event sequence that has occurred in the past. For example, Nelson (1996) provides the narrative of two-year-old Emily who describes a past event as follows "My sleep. Mommy came. And Mommy 'get up get up time go home.' When my slep [sic]... Time to go home. Drink p-water [Perrier]. Yesterday did that. Now Emmy sleeping in regular bed"; for other examples from Emily, see Gerhardt (1989). Emily seems to have a temporally structured representation for an event sequence that happened previously, so she knows when individual components of that event sequence happened relative to each other. However, as discussed above, a mature linear and unified notion of time allows for more than this: it is underpinned by a grasp of the fact that systematic relations exist between any two points in time, even between entirely unrelated events. As mentioned above, these systematic relations have an important property, namely that of transitivity. What we are suggesting 
is that there is currently no convincing evidence that two- to three-year-olds can think about temporal relations in this way.

One challenge to how we have characterized the temporal abilities of children of this age, particularly in terms of assuming that they can only think of time in an event-dependent way, might be thought to come from psycholinguistic studies. Children aged two to three years learn to use temporal adverbs, such as 'now', 'already', 'later', 'when', and 'soon' (Weist, 2014; Weist \& Buczowska 1987; Weist \& Zevenbergen, 2008). They will also use terms such as 'yesterday' and 'tomorrow' (Pawlak et al., 2006; Weist \& Buczowska 1987), although not necessarily accurately. One important issue is whether this indicates that children are using these terms to single out points in time per se, rather than events. If they were, this would suggest that they are not event-dependent in their temporal thinking in the way that we have argued. One way to put this point would be to say that children using these terms may be introducing a temporal context in which to describe events, and, thus, that they are not simply describing the events themselves.

While we agree that the use of these types of terms signals that children's abilities go well beyond those captured in Stage (a), we believe that it does not tell us whether children are actually picking out an event-independent point in time. That is, what these adverbs mean for children when they first start using them might potentially actually be cashed out purely with reference to events themselves, rather than with reference to times as such. So, for example, 'later' could potentially mean 'once certain events have finished', and 'when' might always mean 'simultaneous with a certain event' rather than 'at the point in time at which', and so on. In other words, children may be introducing an event context rather than a temporal context. Nelson (1996) argues for this sort of interpretation of early use of temporal adverbs stating that "the child uses knowledge of event structure as a framework for acquiring and eventually interpreting words coding temporal relations..." (p. 286). Here, what she means is that children learn to use these words in the context of their event representations, and it is only later that the terms acquire their full meaning. She suggests that even terms such as 'yesterday' or 'tomorrow' might initially only be understood in relation to certain events, rather than understood as referring to periods of time in the past and future.

Children's limitations also become clearer by considering that there is no evidence that children of this age are capable of the sort of temporal perspectivetaking that we described in detail in Section 1.3. That is, children may indeed categorize some facts about events as unalterable and some as (at least potentially) alterable [Stage (b)], but may not yet be capable of the sort of temporal perspective-taking that a mature notion of time allows. As far as we are aware, there is no evidence to suggest that children of this age can appreciate that the status of events as unalterable or alterable changes systematically as time passes, and that at different points in time facts about events that were once alterable became no longer so. That is, we do not believe that children of this age are 
capable of the sort of flexible thinking about time that is underpinned by a linear and unidirectional notion of time. We argued in Section 1.3 that such an ability is a hallmark of a mature notion of time, not only because it involves representing the domain of times as linear and unidirectional, but because it involves representing times in an event-independent way. Thinking of a past event as 'once potentially alterable' involves a grasp of the fact that the time the event occurred could have been filled with different possible events; i.e., it involves being able to think about points of time independently of the events that occupy those points in time. Although children of this age are able to talk about some events in the past and future, can use tensed forms appropriately, and use some temporal adverbs, we would argue that this is not sufficient evidence of the understanding we have described.

\subsection{Stage (c): Linear Event-Independent Time (4-5 years)}

In our model, we are suggesting that sometime around four to five years, there is an important shift in how children can think about time, when children start to represent time in a linear and unified way, and become capable of eventindependent thought about times. Children now grasp that there are systematic relations between points in time and realize that as time unfolds things due to happen in the future will become present and then move to the past.

One good reason for assuming that children of this age start to grasp the relations between times comes from studies that ask children about the temporal relations between different events that happened at quite distinct points in time and were not part of a connected sequence. For example, Friedman (1991) showed that four-year-olds can judge the relative order of two unrelated events that occurred in their schools six weeks apart (a lesson on tooth brushing and a demo of how video cameras work). A number of other studies have also shown that fourand five-year-olds make some judgments about the order of unrelated events over extended time periods (Friedman, 2000; Friedman \& Kemp, 1998; Friedman et al., 1995; Hudson \& Mayhew, 2011; McCormack \& Hanley, 2011), although children find it easier to order past than future events (Busby-Grant \& Suddendorf, 2009; McCormack \& Hanley, 2011). Some of these studies used a time line procedure whereby children had to place events at locations on a line stretching back into the past or forward into the future, and although children of this age are not perfect at this task, their above-chance performance suggests that they understood this format (Friedman \& Kemp, 1998; Hudson \& Mayhew, 2011; though see DroitVolet \& Coull, 2015).

A recent study by Tillman and colleagues (2017) provided the most extensive study to date of children's ability to use such a time line. In their study, children were shown a horizontal line, with a vertical line in the middle that marked 'right now', and were asked to mark in the locations of some past (e.g., eating breakfast that morning) and future events (their next birthday), as well as some locations 
corresponding to deictic time words (such as yesterday or next week). By four to five years, but not before, children were able to appropriately place locations in the side of the time line representing the past versus the future, and they also were above chance in ordering the locations appropriately on the time line (e.g., that 'yesterday' is before 'this morning'). Notably, the ability to appropriately locate times in the past versus the future was closely linked to the ability to order locations in time, suggesting that children of this age have developed a coherent linear and unidirectional representation of the domain of time that underpinned both abilities. In summary, findings from a number of different studies including the recent one by Tillman et al. (2017) indicate that, at this age, children understand something important about the relations between points in time.

We have also suggested that event-independent thought about time might be closely linked to counterfactual thought, because thinking counterfactually about a past event indicates that children understand that there is a distinction between the point in time at which an event occurred and the event itself (i.e., something different could have happened, because the point in time at which the event occurred was once in the future and hence still potentially alterable). In fact, there is considerable disagreement amongst cognitive developmental psychologists over when children can think counterfactually (Beck \& Riggs, 2014; Rafetseder \& Perner, 2014). Much of this debate hinges on whether children may be able to answer some types of counterfactual questions without actually engaging in counterfactual thought (Beck, 2016; Rafetseder et al., 2010, 2013).

Beck et al.'s (2006) study examined children's ability to think about both future possibility and counterfactual possibility in a novel way. In their task, children watched as a toy mouse was dropped down into a chute that branched off into two sides. Beck et al. asked children a specific type of counterfactual question: children were asked, after the mouse had fallen down one branch of the chute, whether it could have gone anywhere else (a so-called open counterfactual question). Threeto four-year-olds had difficulties answering this question, but five- to six-year-olds were able to do so. Beck et al. (p. 424) suggest that answering correctly requires that "one appreciates that there was a point in time when either the actual or counterfactual event could have happened". Furthermore, in order to assess the idea that children would consider that different possible outcomes could occur in the future, before dropping the mouse they asked children to put cotton wool mats out to catch it. Children could either put out a single mat or two mats, one for each branch of the chute. Beck et al. argued that if children understood that there could be more than one possible outcome, they should put out mats under both branches. Three- and four-year-olds typically put out only a single mat, and it was not until children were around five years old that they were likely to put out two mats. Given the findings of both these tasks, Beck et al. conclude that it is not until around five years that children can think both about the future and about the present as locations in time at which multiple possible events could occur 
or have occurred. These findings are consistent with the sort of shift in children's understanding of time that we have outlined here.

Being able to think about time in an event-independent way is also important for everyday activities such as some types of planning. Typically, when one is planning, one thinks about not just which actions one might engage in, but also the order in which one should engage in them. One may mentally shuffle these actions about or replace them with other actions in order to try to figure out what the optimal sequence would be. Thus, just as planning a spatial layout may involve an object-independent way of thinking about space - e.g., mentally moving furniture around so that the table goes there and the lamp goes here — so planning may typically involve an event-independent way of thinking about time in which one mentally moves around events to figure out how to obtain the outcome one wants. In their review paper of the research on young children's planning, McCormack and Atance (2011) have argued that there are distinctive developmental changes in children's ability to plan for the future that occur around four to five years. In particular, children start to be able to plan on a variety of different tasks that have in common the requirement to be able to flexibly reason about the temporal relations between steps in a sequence of actions, mentally trying out different possible actions at different steps (e.g., Gauvain \& Rogoff, 1989; Kaller et al., 2008; McColgan \& McCormack, 2008).

Some of these studies can also be interpreted as involving temporal perspective-taking. For example, in McColgan and McCormack's (2008) study, children were told about a character who was visiting a zoo, and wanted to take a picture of a kangaroo. Children had to choose where to leave the character's camera so that she could ensure that she had the camera by the time she got to the kangaroo's cage. Some of the locations were on the path before the kangaroo's cage, and some after. One way of characterizing what children have to do in this task is that they have to realize that by the time the character reaches the cage, the event of picking up the camera, which is now in the future, must be in the past, i.e., they have to be able to reason appropriately about before-and-after relations between points in time and engage in temporal perspective-taking.

We want to emphasize here that this requires an understanding of how causality operates in time. Mature thinkers understand that the passage of time leads to the unfolding of causally linked states of affairs: in terms of an ordered set of points of time in the past $A, B$, and $C$, they can think of the events at $A$ as causally determining a certain outcome at $B$, and events at $B$ as potentially changing that outcome, leading to a different outcome at $C$, and they grasp that these causal relations are not reversible. Elsewhere we have termed this sort of reasoning that involves appreciating the causal significance of temporal order as temporalcausal reasoning (McColgan \& McCormack, 2008; McCormack \& Hoerl 2005, 2007). Note that being able to engage in this form of reasoning goes beyond being able to produce a sequence of causally linked actions to achieve a goal, which 
children can do from an early age. Rather, it requires a reflective ability to reason about temporal-causal relations themselves.

Some studies have directly looked at young children's ability to engage in this sort of reasoning about causality in time, and the general conclusion is that this ability emerges somewhere around four to five years. In examining this ability, it is crucial to distinguish between judgments that involve simply updating one's model of the world sequentially as states of affairs unfold, and actually reasoning about causal relations in time. Updating can allow one to have a model of the world that is sensitive to how the unfolding of a sequence of events has impacted on how things are: it is simply a matter of changing one's representations of how things are systematically as one sequentially acquires pieces of information about states of the world. For example, if a sequence of events A, B, and C occurs, updating simply involves changing one's representation of how things are initially following A, then following B, and then following C. This does not involve reasoning about event order per se. Temporal-causal reasoning is more sophisticated than this, because it involves actually reasoning about how the sequence in which events happened has yielded one outcome rather than another: for example, reasoning that if A happened and then B, this would have led to outcome $\mathrm{X}$ but if $\mathrm{B}$ happened and then A, this would have led to outcome Y. Distinguishing between simple updating and temporal-causal reasoning is not straightforward: it involves decoupling the order in which children find out about events from the order in which the events actually happen, because if children find out about events in the same order in which they actually happen they could well use updating to correctly judge the current state of the world.

The first study to use this approach was that of Povinelli and colleagues (1999). These authors showed children two video clips of a toy being hidden in a box behind their back while they had been playing a game (i.e., children had not seen the hiding events when they occurred). In each video, the child was playing a different game, and the toy was being hidden in a different one of two boxes. The children were shown the two videos together, after they had played both games, but crucially some children were shown the clips in a different temporal order from the one in which the two filmed episodes had actually occurred. Thus, those children had to use their knowledge of the order in which they had played the two games to reason that the toy was now in the location shown in the first video they saw rather than in the second video. It was not until children were five that they were able to do this.

Two subsequent studies by McCormack and Hoerl $(2005,2007)$ also decoupled the order in which children found out about events from actual event order, but did not use a video clip method. In one study (McCormack \& Hoerl, 2007), children were told that two boy dolls always acted in a specific order. The boys then went into a room in a dolls' house that had a red cupboard and a blue cupboard and the door was closed so that children could not see the subsequent events. 
The experimenter narrated to children what was happening, which was that the doll who always went first put a hairbrush in one cupboard (without naming the color), and the other doll got the hairbrush out and put it in a different cupboard. The door to the room was then opened, and the dolls were standing beside the cupboards that they had put the hairbrush in. Children had to use their knowledge about the order in which the dolls acted in order to figure out where the hairbrush was. As in Povinelli et al.'s (1997) study, it was not until children were around five years that they could do this. Using a different procedure, McCormack and Hoerl (2005) found a similar pattern of age effects.

Interestingly, another context in which the order in which children find out about events differs from actual event order is in the context of some types of sentence constructions involving the terms 'before' and 'after'. These terms allow the speaker to decouple order in exactly this way: for example, in the sentence 'Before the girl took off her hat, she took off her coat.', the taking off of the hat happens after the taking off of the coat but is mentioned first. A number of studies have suggested that three- to four-year-olds tend to use an order-of-mention strategy to understand sentences involving 'before' and 'after', meaning that they will typically have difficulty with sentences in which order of mention does not match event order (Blything et al., 2015; French \& Brown, 1977; McCormack \& Hanley, 2011; Trosborg, 1982). One possibility is that children's ability to understand such non-chronological sentences is primarily constrained by their working memory skills (Blything et al., 2015), because of the need to mentally manipulate the sentence content. Alternatively, it could be that young children's comprehension difficulties are part of a larger problem in reasoning about event order. One piece of evidence in favor of the latter possibility is that children's comprehension on such non-chronological sentences is correlated with performance on tasks that involve making judgments or reasoning about event order (McCormack \& Hanley, 2011).

Taken together, these findings suggest that at around four to five years children are able to reason in a new way about how causality operates in time. However, there are only a limited number of studies that have looked at this issue and, moreover, they have all looked at children's reasoning about just two events that occurred over a relatively short time scale of a few seconds to minutes. We believe there is considerable scope to look in more detail at young children's understanding of how causality operates in time, and perhaps to examine how this may be linked to other achievements we have mentioned in this section, such as children's planning abilities, their ability to think counterfactually, their comprehension of temporal terms such as 'before' and 'after' and their ability to make judgments about the order of unrelated temporally distant events. More generally, developmental studies have not directly addressed when children first understand that facts about events in the past are unalterable but that facts about what will happen in the future are potentially alterable (though see Busby Grant \& Suddendorf, 2010) — which we suggest children may grasp at Stage (b) — or 
addressed when children grasp that what is alterable and non-alterable changes as one moves through time and differs from different temporal perspectives, which we suggest is a further developmental achievement.

\subsection{Stage (d):Abstract Time ( $>5$ years)}

We have put in a final stage in our model, which is a stage at which time is assumed to be understood in a completely event-independent way. We are less confident that this constitutes an entirely new stage in children's comprehension of time, but we include this stage to capture the idea that once children master the conventional clock and calendar system, they have a way of describing and thinking about times that makes no reference to events at all. ${ }^{3}$ We suspect that until children have some mastery of such a system, they will inevitably think of times in a way that involves at least considering the events located at those times, even if children can distinguish mentally been an event and its time of occurrence. Moreover, it is likely that learning this system makes the nature of time itself more transparent: as Campbell (2006) argues "When the introduction of a calendar and clock system makes linearity explicit, this is a bold, simple stroke that clarifies our thinking about time." (p. 11). The clock and calendar system provides a powerful new way of singling out temporal locations, and part of its power lies in the fact that it captures both repeated cycles (days, weeks, and years) and particular unique times. Clearly, mastery of this system is only achieved within the context of extensive socialization and typically as a result of direct teaching. Nevertheless, once children acquire this system they arguably possess a way of thinking about time itself that they did not have before.

In fact, full mastery of a clock and calendar system is a very slow developmental achievement (e.g., Friedman, 1977, 1986, 1991, 2000; see Friedman, 1982, 1989; McCormack, 2015, for reviews). Although by age 6 to 7 , children can often report on what day of the week it is and can list the seasons in correct order (Friedman, $1977,1982,1986,1991)$, they can struggle with the cyclical aspect of the system (for example, although they recognize that spring-summer-autumn-winter is the correct order, they may have difficulty judging that autumn-winter-springsummer is also a correct order). Children of 8 to 10 will typically have learnt the correct order of the months of the year, but still have less flexibility than adults in how they make use of the system, and can struggle to make judgments about, for example, which annual holiday or month comes next from the perspective of a different point in the year (Friedman, 1986, 2000). In fact, Friedman (1982, $1986,1989)$ has argued that there is a qualitative shift in adolescence in how

3 Clocks and calendars are calibrated by natural events (and historical ones determine what counts as 'year zero') but they arguably provide those who use them with a way of thinking about times without having to think about those events. 
conventional time is represented. His argument is that children's difficulties using the system flexibly stem from the fact that they initially represent only verbal lists of days and months, which are intrinsically directional and thus cause difficulties when making flexible judgments (e.g., those that involve thinking retrospectively). He suggests that it is only in adolescence that individuals start to use spatialized mental images of conventional time that support more flexible judgments.

\section{Explaining Developmental Change}

As yet, we have said nothing about the nature of the developmental process, and in particular we have not made any claims about how children move from stage to stage. Moreover, we have not considered how the changes in the notion of time described here might be related to any changes in children's acquisition of a concept of duration - the question raised at the start of this paper. There is very little in the way of empirical evidence that can help us address either of these issues, but in this final section we make some remarks about them.

\subsection{Developmental Change, Language, and Social Interaction}

To what extent are children's emerging notions of time dependent on social experience and on language acquisition in particular? Historically, the idea that linguistic experience shapes the conceptualization of time has been a well-known and controversial one, not least because of Whorf's (1941) influential analysis of time in Hopi language. Clearly, the conventional systems for measuring time and locating events in time are culturally specific and are only acquired by children as a result of extensive direct teaching. We take the more difficult issue to be whether acquiring the more basic notions of time that we have highlighted in Stages (a)-(c) of our model is dependent on language and social experience. The most extensive discussion of this issue is provided by Nelson (1996). She argues that through their own experiences, children come to represent familiar event sequences. However, notions of time are only implicit in these representations, and it is only through acquisition of language that these implicit notions become explicit. Moreover, she claims that children's first uses of temporal terms and language are bound up with their event representations and as a result do not have the same meaning as those of adults; it is only gradually through shared discourse with adults about events that children acquire mature temporal notions. Notably, she believes that this is true not only with regard to the acquisition of conventional time systems (such as the clock and calendar system), but also with regard to more fundamental aspects of a notion of time, including the distinction between past, present, and future: "[L]anguage may make salient a type of relation that was not previously apparent in the child's non-linguistic conceptual representations. Present evidence suggests that this may be the case for the tense system, bringing out the distinction between past, present, and future. Prior to acquiring this 
system children may distinguish only between the now and the not-now, or attend only to action relations in the here and how, the living present. In this case, the relation that must be expressed grammatically attunes the child to a relation to which she had previously been indifferent" (p. 289).

The central difficulty in assessing this argument empirically, and other claims about the role of language and social experience in children's acquisition of temporal concepts, is that is extremely difficult to know what sort of concept children have of time without examining their speech or using tasks that require language. Thus, it is difficult if not impossible to contrast what children understand about time before they acquire language with their subsequent understanding. Empirical evidence to support suggestions such as Nelson's takes the form of trying to establish that when children first use (in this instance) tense, they do not seem to use it to mark the same distinctions as adults; this is then interpreted as indicating that they initially lack the distinction between past, present, and future that underpins the adult use of the tensed system. Indeed, we considered some evidence of this sort in Section 2.2.

While we do not necessarily agree with all aspects of Nelson's (1996) account, we suspect that she may be correct in assuming that acquiring fundamental temporal notions may be something that hinges on acquiring language and engaging in shared discourse. Elsewhere, we have argued that engaging in shared reminiscence about the past (and shared discussion about the future) with adults may be critical in children acquiring a linear, unidirectional notion of time that is underpinned by a grasp of how causation operates in time (Hoerl, 2007; Hoerl \& McCormack, 2005; McCormack, 2015; see also Welch-Ross, 2001). The suggestion is that when parents and caregivers engage in talk about events at other times with children, they are essentially scaffolding children to begin to take different temporal perspectives on events. For example, by adopting a different temporal perspective on an event that is now in the past, parents can help children understand that the very same event once had not yet unfolded and was still in the future. Similarly, it can illustrate how what did happen was both constrained by what had happened previously and also determined by what choices were made at the time. In this way, children may start to understand the causal connections between events located at different points in time. While there is little empirical evidence to directly support the idea that such interactions are critical for acquisition of children's notion of time, there is good evidence to indicate that these interactions support the development of children's ability to form their own coherent and structured narratives about their personal past and future and to use temporal terms appropriately (Hudson, 2006; Nelson \& Fivush, 2004).

\subsection{A Single Process or Multiple Ones?}

Finally, we began this article by distinguishing between two ways in which time features in people's mental lives - time as a dimension of things that unfold in 
time and time as a framework within which such things can be located - and have focused on the development of the second. Once children have mastered the clock and calendar system, we can be confident that they have integrated both of these aspects of the concept of time. However, we do not know whether these really are separable aspects of a concept of time that develop independently, and, if they are separable, how this integration is achieved. One possibility is that they are acquired in entirely separate developmental processes, and they become properly integrated only when children start to learn the clock and calendar system. The alternative is that their acquisition depends on some of the same cognitive achievements and socio-cultural experiences. There is no empirical evidence that we are aware of that would help us decide between these alternatives.

We note, though, that whilst this paper has in particularly focused on the question of the emergence of a capacity for event-independent thought in the context of children's understanding of time as a framework, there is an important parallel issue regarding children's understanding of time as a dimension, namely when children begin to think of duration in an event-independent way. That is, when can children think of a period of time as quantifiable, independent of whatever events actually occur during that time period? Indeed, answering this question is part of what the Piagetian research programme attempted to do, although by the questionable method of looking at when duration judgments were unaffected by other stimulus dimensions. Perhaps a better way to try to answer this question may be to look at when children begin to count in order to measure time intervals, which could be interpreted as evidence that they understand that duration is quantifiable. Although children often do not count spontaneously before around 8 years (Pouthas et al., 1995; Wilkening et al., 1987), children as young as 5 can be encouraged to count and it improves the accuracy of their duration judgments (Clement \& Droit-Volet, 2006).

Other studies have shown that by the time children are five they can use some sort of metric to make judgments about the durations of events. Friedman (1990) showed that five-year-olds can use a rudimentary scale involving sandtimers of different sizes in order to make judgments of the durations of every day events, suggesting that they have a way of thinking about duration - i.e., a single metric - that is uniform across different event types. More recently, Tillman and Barner (2015) asked children to make judgments about the relative durations of familiar events by indicating on a time line how long they thought the events lasted for, with one end of the time line being described as very short and the other end as very long. Using this technique, five-year-olds were able to make some discriminations in this way between familiar events of different durations, even though they showed poor knowledge of the durations of periods of time described using time words associated with the conventional clock and calendar system (seconds, minutes, hours, and days). Indeed, perhaps unsurprisingly, a proper grasp of the durations of periods of time corresponding to conventional time words seems 
to be closely linked to children's numerical skills, and continues to develop over middle childhood (Labrell et al., 2016).

Further research by Droit-Volet using a quite different technique also sheds light on the issue of when children have a concept of duration. Droit-Volet and Rattat (1999) trained three- and five-year-olds to make a cartoon picture appear by pressing a rubber squeezer for $5 \mathrm{~s}$; children of both age groups were able to learn to do this, consistent with other studies suggesting that three-year-olds can appropriately adjust their actions to demonstrate sensitivity to event duration (Droit-Volet \& Coull, 2016; Rattat \& Droit-Volet, 2007). They then used a transfer task in which children had to perform a different action but of the same duration of $5 \mathrm{~s}$ in order to see the cartoon picture. They found that while five-year-olds were able to transfer knowledge about appropriate duration to a different action, threeyear-olds were unable to do so. They argued that this suggests that by five years (but not before) children can not only time their actions appropriately, but they also have a concept of duration that is sufficiently abstracted from the specific events filling a duration to allow them to succeed on the transfer test.

Taken together, these studies suggest that by five years, children have some concept of duration that is event-independent, i.e., that they understand that events last for a certain amount of time, and that even for periods filled with different types of events it is possible to compare the amount of time that they take. Rattat and Droit-Volet (2007, p. 282) refer to this as a concept of "homogeneous time abstracted from events". We note that the age in question (around five years) is around the same age as we suggest children reach Stage (c) of our model, i.e., when they begin to think about the domain of time in which events can be located in an event-independent way. Both conceptual achievements - being able to represent the domain of time in a linear, event-independent way, and possessing a concept of duration - pave the way for children to begin to acquire knowledge of the clock and calendar system, although this learning process takes a long time for children to complete.

As we stated above, there is little empirical evidence that allows us to judge whether acquiring these two notions of time depends on separable processes. However, we note that there is a hint from Tillman et al.'s (2017) recent study of children's understanding of deictic terms that while four- to five-year-olds can represent time as a linear domain of ordered locations, they may not yet properly integrate this with knowledge about the lengths of periods of time. Children of this age were able to appropriately order temporal locations on a time line relative to each other (e.g., place 'yesterday' to the right of 'last year' or 'next week' to the left of 'next year'), but their placements suggested they were much poorer at judging the relative durations of the period of time between the event and the current moment in time (e.g., that 'next year' is considerably farther from the present than 'tomorrow'). This suggests that while they may have some grasp of the ordering of times in the past and future, they find it hard to put this together with 
information about how much time there is between the present and these other times. Clearly, these findings address primarily children's understanding of temporal terms (some of which are associated with the conventional calendar system, and a proper grasp of which must require numerical skills), so it is difficult to judge to what extent this reflects conceptual difficulties. A promising line of psycholinguistic research, though, would be to examine relations between children's understanding of temporal terms referring to duration and children's understanding of deictic temporal terms.

\section{Conclusion}

We have proposed a stage-like developmental model of the acquisition of temporal concepts, but, as we acknowledge above, this model is still speculative. As we have pointed out, there is as yet no agreed set of experimental tasks used to study children's temporal concepts. In proposing our model we have drawn in part on existing literature in psycholinguistics, much of which has looked at children's spontaneous use of tensed or temporal language. While this is indeed a rich source of data, we suspect that until there are established empirical paradigms testing the specific aspects of children's understanding of time that we have detailed in this paper, this area of cognitive development will remain relatively neglected. And part of the difficulty in coming up with such paradigms lies in designing tasks that do not simply test children's understanding of language itself.

We have focused in this paper on children's emerging understanding of time as a domain in which events can be located, but we have also mentioned the importance of studying children's acquisition of the concept of duration. In looking across both aspects of temporal cognition (time as a dimension, time as framework), what needs to develop is an event-independent way of thinking about time. This is the central developmental achievement, and, as we have argued, it is one that is likely to happen in degrees.

\section{Acknowledgments}

This research was supported by ESRC grants ES/N01281X/1 and ES/N000900/1, and by the AHRC grant AH/P00217X/1.

\section{References}

Antinucci, F., \& Miller, R. (1976). How children talk about what happened.J. Child Lang., 3, 167-189. Aveni, A. (2000). Empires of Time. London, UK: Tauris.

Bauer, P. J., \& Wewerka, S. S. (1995). One-to two-year-olds' recall of events: The more expressed, the more impressed. J. Exp. Child Psychol., 59, 475-496.

Bauer, P. J., Hertsgaard, L. A., \& Dow, G. A. (1994). After 8 months have passed: Long-term recall of events by 1- to 2-year-old children. Memory, 2, 353-382. 
Beck, S. R. (2016). Why what is counterfactual really matters: A response to Weisberg and Gopnik, Cogn Sci, 40, 253-256.

Beck, S. R., \& Riggs, K. J. (2014). Developing thoughts about what might have been. Child Dev. Perspect., 8, 175-179.

Beck, S. R., Robinson, E. J., Carroll, D. J., \& Apperly, I. A. (2006). Children's thinking about counterfactuals and future hypotheticals as possibilities. Child Dev, 77, 413-426.

Bender, A., \& Beller, S. (2014). Mapping spatial frames of reference onto time: A review of theoretical accounts and empirical findings. Cognition, 132, 342-382.

Bennett, J. (1989). Rationality. Indianapolis, IN, USA: Hackett:

Blything, L. P., Davies, R., \& Cain, K. (2015). Young children's comprehension of temporal relations in complex sentences: The influence of memory on performance. Child Dev., 86, 1922-1934.

Boroditsky, L., \& Gaby, A. (2010). Remembrances of times East: Absolute spatial representations of time in an Australian aboriginal community. Psychol. Sci., 21, 1635-1639.

Boroditsky, L., Fuhrman, O., \& McCormick, K. (2011). Do English and Mandarin speakers think about time differently? Cognition, 118, 123-129.

Brannon, E. M., Roussel, L. W., Meck, W. H., \& Woldorff, M. (2004). Timing in the baby brain. Cogn Brain Res, 21, 227-233.

Bronckart, J. P., \& Sinclair, H. (1973). Time, tense, and aspect. Cognition, 2, 107-130.

Busby Grant, J., \& Suddendorf, T. (2009). Preschoolers begin to differentiate the times of events from throughout the lifespan. Eur. J. Dev. Psychol., 6, 746-762.

Busby Grant, J., \& Suddendorf, T. (2010). Young children's ability to distinguish past and future changes in physical and mental states. Br.J. Dev. Psychol., 28, 853-870.

Campbell, J. (1995). Past, Space, and Self. Cambridge, MA, USA: MIT Press.

Campbell, J. (1997). The structure of time in autobiographical memory. Eur.J. Philos., 5, 105-118.

Campbell, J. (2006). Ordinary thinking about time. In F. Stadler \& M. Stoeltzner (Eds), Time and History: Proceedings of the 28th International Wittgenstein Symposium (pp. 1-12). Frankfurt, Germany: Ontos Verlag.

Clement, A., \& Droit-Volet, S. (2006). Counting in a time discrimination task in children and adults. Behav. Processes, 71, 164-171.

Colombo, J., \& Richman, W. A. (2002). Infant timekeeping: Attention and temporal estimation in 4-month-olds. Psychol. Sci., 13, 475-479.

Cromer, R. F. (1971). The development of the ability to decenter in time. Br. J. Psychol., 62, 353-365.

Dainton, B. (2010). Time and Space. Durham, UK: Acumen.

de Hevia, M. D., Izard, V., Coubart, A., Spelke, E. S., \& Streri, A. (2014). Representations of space, time, and number in neonates. Proc. Natl Acad. Sci. USA, 111, 4809-4813.

Droit-Volet, S. (2002). Scalar timing in temporal generalization in children with short and long stimulus durations. Q.J. Exp. Psychol. A, 55, 1193-1209.

Droit-Volet, S. (2013). Time perception in children: A neurodevelopmental approach. Neuropsychologia, 51, 220-234.

Droit-Volet, S. (2016). Development of time. Curr. Opin. Behav. Sci., 8, 102-109.

Droit-Volet, S., \& Coull, J. (2015). The developmental emergence of the mental time-line: Spatial and numerical distortion of time judgement. PloS One, 10, e0130465. doi: 10.1371/journal. pone.0130465.

Droit-Volet, S., \& Coull, J. T. (2016). Distinct developmental trajectories for explicit and implicit timing. J. Exp. Child Psychol., 150, 141-154. 
Droit-Volet, S., \& Rattat, A. C. (1999). Are time and action dissociated in young children's time estimation? Cogn. Dev., 14, 573-595.

Fivush, R., \& Nelson, K. (2004). Culture and language in the emergence of autobiographical memory. Psychol. Sci., 15, 573-577.

French, L. A., \& Brown, A. L. (1977). Comprehension of before and after in logical and arbitrary sequences. J. Child Lang., 4, 247-256.

Friedman, W. J. (1977). Development of children's understanding of cyclic aspects of time. Child Dev., 48, 1593-1599.

Friedman, W. J. (1982). Conventional time concepts and children's structuring of time. In W. J. Friedman (Ed.), The Developmental Psychology of Time (pp. 171-208). New York, NY, USA: Academic Press.

Friedman, W. J. (1986). The development of children's knowledge of temporal structure. Child Dev., $57,1386-1400$.

Friedman, W.J. (1989). The representation of temporal structure in children, adolescents, and adults. In I. Levin \& D. Zakay (Eds), Time and Human Cognition: A Life-Span Perspective (pp. 259-304). Amsterdam, Netherlands: North Holland.

Friedman, W. J. (1990). Children's representations of the pattern of daily activities. Child Dev., 61, 1399-1412.

Friedman, W. J. (1991). The development of children's memory for the time of past events. Child Dev., 62, 139-155.

Friedman, W. J. (2000). The development of children's knowledge of the times of future events. Child Dev., 71, 913-932.

Friedman, W. J., \& Kemp, S. (1998). The effects of elapsed time and retrieval on young children's judgments of the temporal distances of past events. Cogn. Dev., 13, 335-367.

Friedman, W. J., Gardner, A. G., \& Zubin, N. R. E. (1995). Children's comparisons of the recency of 2 events from the past year. Child Dev., 66, 970-983.

Fuhrman, O., \& Boroditsky, L. (2010). Cross-cultural differences in mental representations of time: Evidence from an implicit nonlinguistic task. Cogn. Sci., 34, 1430-1451.

Gauvain, M., \& Rogoff, B. (1989). Collaborative problem solving and children's planning skills. Dev. Psychol., 25, 139-151.

Gell, A. (1992). The Anthropology of Time. Oxford, UK: Berg.

Gerhardt, J. (1989). Monologue as a speech genre. In K. Nelson (Ed.), Narratives from the Crib (pp. 171-230). Cambridge, MA, USA: Harvard University Press.

Harner, L. (1982). Talking about the past and the future. In W. J. Friedman (Ed.), The Developmental Psychology of Time (pp. 141-170). New York, NY, USA: Academic Press.

Hassig, R. (2001). Time, History, and Belief in Aztec and Colonial Mexico. Austin, TX, USA: University of Texas Press.

Hayne, H., Gross, J., McNamee, S., Fitzgibbon, O., \& Tustin, K. (2011). Episodic memory and episodic foresight in 3- and 5-year-old children. Cogn. Dev., 26, 343-355.

Hoerl, C. (2007). Episodic memory, autobiographical memory, narrative: On three key notions in current approaches to memory development. Philos. Psychol., 20, 621-640.

Hoerl, C. (2008). On being stuck in time. Phenomenol. Cogn. Sci., 7, 485-500.

Hoerl, C., \& McCormack, T. (2005). Joint reminiscing as joint attention to the past. In N. Eilan, C. Hoerl, T. McCormack, \& J. Roessler (Eds), Joint Attention: Communication and Other Minds (pp. 260-286). Oxford, UK: Oxford University Press. 
Hudson, J. A. (2006). The development of future time concepts through mother-child conversation. Merrill Palmer Q., 52, 70-95.

Hudson, J. A., \& Mayhew, E. M. Y. (2011). Children's temporal judgments for autobiographical past and future events. Cogn. Dev., 26, 331-342.

Kaller, C. P., Rahm, B., Spreer, J., Mader, I., \& Unterrainer, J. M. (2008). Thinking around the corner: The development of planning abilities. Brain Cogn., 67, 360-370.

Labrell, F., Mikaeloff, Y., Perdry, H., and Dellatolas, G. (2016). Time knowledge acquisition in children aged 6 to 11 years and its relationship with numerical skills. J. Exp. Child Psychol., 143, 1-13.

Le Guen, O., Balam, P., \& Ildefonsa, L. (2012). No metaphorical timeline in gesture and cognition among Yucatec Mayas. Front. Psychol., 3, 271. doi: 10.3389/fpsyg.2012.00271.

Levin, I. (1982). The nature and development of time concepts in children: The effect of interfering cues. In W. J. Friedman (Ed.), The Developmental Psychology of Time (pp. 47-86). New York, NY, USA: Academic Press.

Levin, I. (1992). The development of the concept of time in children: An integrative model. In F. Macar, V. Pouthas, \& W. J. Friedman (Eds), Time, Action, and Cognition: Towards Bridging the Gap (pp. 13-32). Dodrecht, The Netherlands: Kluwer.

Lourenco, S. F., \& Longo, M. R. (2010). General magnitude representation in human infants. Psychol. Sci., 21, 873-881.

Matthews, W. J., \& Meck, W. H. (2016). Temporal cognition: Connecting subjective time to perception, attention, and memory. Psychol. Bull., 142, 865-907.

McColgan, K. L., \& McCormack, T. (2008). Searching and planning: Young children's reasoning about past and future event sequences. Child Dev., 79, 1477-1497.

McCormack, T. (2001). Attributing episodic memory to animals and children. In C. Hoerl \& T. McCormack (Eds) Time and Memory: Issues in Philosophy and Psychology (pp. 285-314). Oxford, UK: Oxford University Press.

McCormack, T. (2014). Three types of temporal perspective: Characterizing developmental changes in temporal thought. Ann. N. Y. Acad. Sci., 1326, 82-89.

McCormack, T. (2015). The development of temporal cognition. In L. Liben \& U. Müller (Eds), Handbook of Child Psychology and Developmental Science, Vol. 2, Cognitive Processes, 7th ed. (pp. 624-670). New York, NY, USA: Wiley.

McCormack, T., \& Atance, C. M. (2011). Planning in young children: A review and synthesis. Dev. Rev., 31, 1-31.

McCormack, T., \& Hanley, M. (2011). Children's reasoning about the temporal order of past and future events. Cogn. Dev., 26, 299-314.

McCormack, T., \& Hoerl, C. (1999). Memory and temporal perspective: The role of temporal frameworks in memory development. Dev. Rev., 19, 154-182.

McCormack, T., \& Hoerl, C. (2005). Children's reasoning about the causal significance of the temporal order of events. Dev. Psychol., 41, 54-63.

McCormack, T., \& Hoerl, C. (2007). Young children's reasoning about the order of past events. J. Exp. Child Psychol., 98, 168-183.

McCormack, T., \& Hoerl, C. (2011). Tool use, planning, and future thinking in children and animals. In T. McCormack, C. Hoerl, \& S. Butterfill (Eds), Tool Use and Causal Cognition (pp. 129-147). Oxford, UK: Oxford University Press.

Meltzoff, A. N. (1995). Understanding the intentions of others: re-enactment of intended acts by 18-month-old children. Dev. Psychol., 31, 838-850. 
Nazzi, T., Bertoncini, J., \& Mehler, J. (1998). Language discrimination by newborns: Towards an understanding of the role of rhythm. J. Exp. Psychol. Hum. Percept. Perform., 24, 756-766.

Nelson, K. A. (1996). Language in Cognitive Development. Cambridge, UK: Cambridge University Press.

Nelson, K. D., \& Fivush, R. (2004). The emergence of autobiographical memory: A social cultural developmental theory. Psychol. Rev., 111, 486-511.

Núñez, R. E., \& Sweetser, E. (2006). With the future behind them: Convergent evidence from Aymara language and gesture in the crosslinguistic comparison of spatial construals of time. Cogn. Sci., 30, 401-450.

Pawlak, A., Oehlrich, J. S., \& Weist, R. M. (2006). Reference time in child English and Polish. First Lang., 26, 281-297.

Peterson, C. (2002). Children's long-term memory for autobiographical events. Dev. Rev., 22, 370-402.

Peterson, C., \& Rideout, R. (1998). Memory for medical emergencies experienced by 1- and 2-yearolds. Dev. Psychol., 34, 1059-1072.

Piaget, J. (1969). The Child's Conception of Time. London, UK: Routledge and Keegan Paul.

Pouthas, V., Paindorge, B., \& Jacquet, A. Y. (1995). Learning to measure time: Studies with 4- to 7-year-old. Annee Psychol., 95, 593-619.

Povinelli, D. J., Landry, A. M., Theall, L. A., Clark, B. R., \& Castille, C. M. (1999). Development of young children's understanding that the recent past is causally bound to the present. Dev. Psychol., 35, 1426-1439.

Quon, E., \& Atance, C. M. (2010). A comparison of preschoolers' memory, knowledge, and anticipation of events. J. Cogn. Dev., 11, 37-60.

Rafetseder, E., \& Perner, J. (2014). Counterfactual reasoning: sharpening conceptual distinctions in developmental studies. Child Dev. Perspect., 8, 54-58.

Rafetseder, E., Cristi-Vargas, R., \& Perner, J. (2010). Counterfactual reasoning: Developing a sense of "nearest possible world". Child Dev., 81, 376-389.

Rafetseder, E., Schwitalla, M., \& Perner, J. (2013). Counterfactual reasoning: From childhood to adulthood. J. Exp. Child Psychol., 114, 389-404.

Rattat, A. C., \& Droit-Volet, S. (2007). Implicit long-term memory for duration in young children. Eur. J. Cogn. Psychol., 19, 271-285.

Richards, D. D. (1982). Children's time concepts: Going the distance. In W. J. Friedman (Ed.), The Developmental Psychology of Time (pp. 13-26). New York, NY, USA: Academic Press.

Sachs, J. (1983). Talking about the there and then: The emergence of displaced reference in parent-child discourse. In K. E. Nelson (Ed.), Children's Language, Vol. 4 (pp. 1-28). Hillsdale, NJ, USA: Erlbaum.

Shirai, Y., \& Andersen, R. W. (1995). The acquisition of tense-aspect morphology: A prototype account. Language, 71, 743-762.

Shirai, Y., \& Miyata, S. (2006). Does past tense marking indicate the acquisition of the concept of temporal displacement in children's cognitive development? First Lang., 26, 45-66.

Siegler, R. S., \& Richards, D. D. (1979). Development of time, speed, and distance concepts. Dev. Psychol., 15, 288-298.

Sinha, C., \& Gärdenfors, P. (2014). Time, space, and events in language and cognition: A comparative view. Ann. N. Y. Acad. Sci., 1326, 72-81.

Srinivasan, M., \& Carey, S. (2010). The long and the short of it: On the nature and origin of functional overlap between representations of space and time. Cognition, 116, 217-241. 
Tillman, K. A., \& Barner, D. (2015). Learning the language of time: Children's acquisition of duration words. Cogn. Psychol., 78, 57-77.

Tillman, K. A., Marghetis, T., Barner, D., \& Srinivasan, M. (2017). Today is tomorrow's yesterday: Children's acquisition of deictic time words. Cogn. Psychol., 92, 87-100.

Trosborg, A. (1982). Children's comprehension of before and after reinvestigated. J. Child Lang., 9, 381-402.

vanMarle, K., \& Wynn, K. (2006). Six-month-old infants use analog magnitudes to represent duration. Dev. Sci., 9, F41-F49.

Weist, R. M. (1989). Time concepts in language and thought: Filling the Piagetian void between two and five years. In I. Levin \& D. Zakay (Eds), Time and Human Cognition: A Life-Span Perspective (pp. 63-118). Amsterdam, Netherlands: North Holland.

Weist, R. M. (2014). Developing temporal systems. Psychol. Lang. Commun., 18, 126-142.

Weist, R. M., \& Buczowska, E. (1987). The emergence of temporal adverbs in child Polish. First Lang., 7, 217-229.

Weist, R. M., \& Zevenbergen, A. A. (2008). Autobiographical memory and past time reference. Lang. Learn. Dev., 4, 291-308.

Welch-Ross, M. (2001). Personalizing the temporally extended self: Evaluative self-awareness and the development of autobiographical memory. In C. Moore, \& K. Lemmon (Eds), The Self in Time (pp. 97-120). Mahwah, NJ, USA: Erlbaum.

Whorf, B. L. (1941). The relation of habitual thought and behavior to language. In: L. Spier, A. I. Hallowell, \& S. S. Newman (Eds), Language, Culture and Personality: Essays in Memory of Edward Sapir (pp. 75-93). Menasha, WI, USA: Sapir Memorial Publication Fund.

Wilkening, F. (1982). Children's knowledge about time, distance, and velocity interrelations. In W. J. Friedman (Ed.), The Developmental Psychology of Time (pp. 87-112). New York, NY, USA: Academic Press.

Wilkening, F., Levin, I., \& Druyan, S. (1987). Children's counting strategies for time quantification and integration. Dev. Psychol., 23, 823-883. 